\title{
ON THE ROSENTHAL COMPACTA AND ANALYTIC SETS
}

\author{
ADAM KRAWCZYK
}

(Communicated by Andreas R. Blass)

\begin{abstract}
We consider pointwise convergence in a separable Rosenthal compactum. The main result is that if $X \subset \mathbf{R}^{\omega^{\omega}}$ is a Rosenthal compactum, $Y \subset X$ is countable dense and $x \in X$, then the following are equivalent :

(i) $Y$ has a countable base at $x$.

(ii) $\left\{\left(y_{i}\right) \in Y^{\omega}: \lim _{i \rightarrow \infty} y_{i}=x\right\}$ is analytic, when $Y$ has the discrete topology.
\end{abstract}

\section{INTRODUCTION}

In the paper we will deal mainly with analytic spaces, i.e., continuous images of the Baire space. The class of analytic sets will be denoted by $\Sigma_{1}^{1}$. When $X, Y$ are topological spaces then, throughout this paper, $X^{Y}$ is the space of all functions from $Y$ to $X$ with pointwise convergence topology. We will follow the standard set theoretical notation, so an ordinal is equal to the set of its predecessors. In particular, the set of natural numbers will be denoted by $\omega$, and if $n \in \omega$, then $n=\{0, \ldots, n-1\}$. The only topology on $\omega$ which will be considered is the discrete one, so $\omega^{\omega}$ is the Baire space.

Among compact subspaces of $\mathbf{R}^{\omega^{\omega}}$ particularly nice are Rosenthal compacta, i.e., compact subspaces $X \subset \mathbf{R}^{\omega^{\omega}}$ such that every element of $X$ is a pointwise limit of continuous functions (see [R]). There are separable and not first countable Rosenthal compacta. Many examples of such compacta are constructed in [Ma]. However in the beautiful paper [BFT] Bourgain, Fremlin, and Talagrand proved that a Rosenthal compactum is a Frechet space.

The key to our paper is the notion of bisequentiality (see [P1, Definition 3.D.1]).

Definition 1. The space $X$ is bisequential at a point $x \in X$ if every ultrafilter $p$ in $X$ converging to $x$ contains a sequence of sets converging to $x$.

Pol in [P1] improved the result of Bourgain, Fremlin, Talagrand and obtained the following:

Received by the editors June 12, 1990 and, in revised form, January 16, 1991.

1991 Mathematics Subject Classification. Primary 04A15, 54H05.

The final version of this paper was prepared when the author visited Forschungsintitut für Mathematik ETH Zürich; the author would like to express his gratitude to the Institute for its kind hospitality. 
Theorem 1 (Pol). A separable Rosenthal compactum is bisequential.

The main theorem we are going to prove is the following:

Theorem 2. Assume that $X$ is a Rosenthal compactum, $Y \subset X$ is countable and dense, $x \in X$. Then the following are equivalent :

(i) $Y$ has a countable base at $x$.

(ii) $\left\{\left(y_{i}\right) \in Y^{\omega}: \lim _{i \rightarrow \infty} y_{i}=x\right\}$ is $\mathbf{F}_{\sigma \delta}$.

(iii) $\left\{\left(y_{i}\right) \in Y^{\omega}: \lim _{i \rightarrow \infty} y_{i}=x\right\}$ is analytic.

In (ii) and (iii), $Y$ is considered with the discrete topology.

It has to be pointed out that the set $\left\{\left(y_{i}\right) \in Y^{\omega}: \lim _{i \rightarrow \infty} y_{i}=x\right\}$ is always coanalytic. One can check it easily by direct computation, as in the proof of the theorem in $\S 3$.

The paper is organized as follows: In $\S 2$ we will prove three technical lemmas, which allow us derive in $\S 3$ the theorem and other corollaries.

\section{LEMMAS}

In this section we will work with families of subsets of $\omega$ or $\omega^{<\omega}=\bigcup_{n \in \omega} \omega^{n}$. We present notation and definitions for $\omega$, but all can be applied to $\omega^{<\omega}$ as well.

In the theory of filters on $\omega$ there is a strong trend to denote everything by *. We will follow that nasty tendency: so $A \subset^{*} B$ means $A \backslash B$ is finite; $A={ }^{*} B$ stands for $A \subset^{*} B$ and $B \subset^{*} A ; \omega^{*}=\beta \omega \backslash \omega$ is the set of non-principal ultrafilters on $\omega$; but if $\mathscr{F} \subset \mathscr{P}(\omega)$ then $\mathscr{F}^{*}=\{\omega \backslash A: A \in \mathscr{F}\}$. Hence if $p \in \omega^{*}$ then $p^{*}$ is the maximal ideal on $\omega$ dual to $p, p^{* *}=p$ and $\forall \mathscr{F} \in \mathscr{P}(\omega) \exists p \in \omega^{*} \mathscr{F}^{*} \subset^{*} p^{*}$ is the following (false) statement: For every family $\mathscr{F}$ of subsets of $\omega$ there exists $I-$ a maximal nonprincipal ideal on $\omega$ such that $\{B: \omega \backslash B \in \mathscr{F}\} \backslash I$ is finite.

Definition 2. Let $\mathscr{F}, \mathscr{G} \subset \mathscr{P}(\omega)$. Then

- $\widehat{\mathscr{F}}=\left\{B \subset \omega: \forall A \in \mathscr{F} B \cap A={ }^{*} \emptyset\right\}$.

- $\mathscr{F}$ is countably $\mathscr{G}$-generated if there exists $\left\{A_{n}: n \in \omega\right\} \subset \mathscr{G}$ such that $B \subset A_{0} \cup \cdots \cup A_{k}$ for all $B \in \mathscr{F}$, and some $k \in \omega$.

- $\mathscr{F}$ is bisequential if for every $p \in \beta \omega$ with $\mathscr{F} \subset p^{*}, \mathscr{F}$ is countably $p^{*}$-generated .

Whenever $\mathscr{F}$ is an ideal then the definition of bisequentiality is closely connected with that from the introduction. Details become transparent in the next section.

The following lemma contains the key combinatorial part of the argument.

Lemma 1. Assume $I \subset \mathscr{P}(\omega)$ is bisequential and $\mathscr{F} \subset I$ is not countably I-generated. Then there exists a pairwise disjoint family $\mathscr{A}=\left\{A_{n}: n \in \omega\right\}$ such that I is $\mathscr{A}$-generated and $\mathscr{F} \cap \mathscr{P}\left(A_{n}\right)$ is not countably I-generated for every $n \in \omega$.

Proof. Consider $I^{\prime}=\{C \subset \omega: \mathscr{F} \cap \mathscr{P}(C)$ is countably $I$-generated $\}$. By definition $I \subset I^{\prime}$, and by assumption that $\mathscr{F}$ is not countably $I$-generated we get $\omega \notin I^{\prime}$. It is easy to see that $I^{\prime}$ is an ideal. Let $p \in \beta \omega$ be such that $I^{\prime} \subset p^{*}$. 
Then, by bisequentiality of $I$ we can find a family $\left\{D_{n}: n \in \omega\right\} \subset p^{*}$, such that $I$ is $\left\{D_{n}: n \in \omega\right\}$-generated. Without loss of generality one can assume that $D_{n}$ 's are pairwise disjoint. Put $N=\left\{n \in \omega: D_{n} \in I^{\prime}\right\}$.

Claim 1. $\bigcup_{n \in N} D_{n} \in I^{\prime}$.

Proof of the Claim. By definition of $I^{\prime}$ for every $n \in N$ there exists $\left\{E_{k}^{n}\right.$ : $k \in \omega\} \subset I$, such that $\mathscr{F} \cap \mathscr{P}\left(D_{n}\right)$ is $\left\{E_{k}^{n}: k \in \omega\right\}$-generated. But then $\mathscr{F} \cap \mathscr{P}\left(\bigcup_{n \in N} D_{n}\right)$ is $\left(\bigcup_{n \in N}\left\{E_{k}^{n}: k \in \omega\right\}\right)$-generated.

Indeed, let $C \in \mathscr{F} \cap \mathscr{P}\left(\bigcup_{n \in N} D_{n}\right)$. Then there exists $m \in \omega$ such that $C \subset$ $D_{0} \cup \cdots \cup D_{m-1}$. Since $C \subset \bigcup_{n \in N} D_{n}$ and $D_{n}$ 's are disjoint, $C \subset \bigcup_{n \in N \cap m} D_{n}$. Now, for every $n \in m \cap D$, we can find $k_{n} \in \omega$ such that $C \cap D_{n} \subset E_{0}^{n} \cup \cdots \cup$ $E_{k^{n}}^{n}$; hence $C \subset \bigcup_{n \in N \cap m} E_{0}^{n} \cup \cdots \cup E_{k_{n}}^{n}$ and we are done.

We will show now that $\omega \backslash N$ is infinite. Assume for a contradiction that it is not the case. Then $\bigcup_{n \notin N} D_{n} \in p^{*}, \bigcup_{n \in N} D_{n} \in I^{\prime} \subset p^{*}$ and, by properties of $D_{n}$ 's and definition of $I^{\prime}$, we get $\left(\omega \backslash \bigcup_{n \in \omega} D_{n}\right) \subset(\omega \backslash \bigcup \mathscr{F}) \in I^{\prime} \subset p^{*}$. This implies $\omega \in p^{*}$, a contradiction.

Now let $\left\{k_{n}: n \in \omega\right\}$ be a 1-1 enumeration of $\omega \backslash N$. Put $A_{0}=\bigcup_{n \in N} D_{n} \cup$ $D_{k_{0}}, A_{n}=D_{k_{n}}$ for $n>0$. Obviously $\left\{A_{n}: n \in \omega\right\}$ satisfies the conclusion of the lemma.

For $s \in \omega^{<\omega}$ let $\hat{s}$ denote the set $\left\{t \in \omega^{<\omega}: s \subset t\right\}$. Lemma 2 contains the topological part of the argument.

Lemma 2. Assume $I \in \Sigma_{1}^{1}$ is a bisequential and not countably I-generated ideal on $\omega$. Then there exists $T: \omega^{<\omega} \stackrel{1-1}{\longrightarrow} \omega$ such that for $J=T^{-1}(I)$ the following holds:

(i) $\forall f \in \omega\{f \mid n: n \in \omega\} \in J$.

(ii) $\forall B \in J \forall n \in \omega \exists s_{0}, \ldots, s_{k} \in \omega^{n} B \subset^{*} \hat{s}_{0} \cup \cdots \cup \hat{s}_{k}$.

Proof. Let $\varphi: \omega^{\omega} \stackrel{\text { onto }}{\longrightarrow} I$ be continuous. By induction on length of $s(\mathrm{lh} s)$ we construct $F_{s}$ and $A_{s}$ such that when $T(s)=\min A_{s}$, the following conditions will hold:

(a) $s \supset t \Rightarrow A_{s} \subset A_{t} \backslash\{T(t)\} \subset \omega$.

(b) $s, t \in \omega^{n}, s \neq t \Rightarrow A_{s} \cap A_{t}=\emptyset$.

(c) $F_{s}$ is a closed subset of $\omega^{\omega}$.

(d) The diameter of $F_{s}$ is less than $2^{-\mathrm{lh} s}$.

(e) $s \supset t \Rightarrow F_{s} \subset F_{t}$.

(f) $\varphi\left(F_{s}\right) \subset \mathscr{F}_{s}$, where $B \in \mathscr{F}_{s}$ if and only if $\{T(s \mid 0), \ldots, T(s \mid(\operatorname{lh} s-1))\}$ $\subset B \subset A_{s} \cup\{T(s \mid 0), \ldots, T(s \mid(\operatorname{lh} s-1))\}$.

(g) $\varphi\left(F_{s}\right)$ is not countably $I$-generated .

(h) $I \cap \mathscr{P}\left(A_{s} \backslash\{T(s)\}\right)$ is $\left\{A_{s^{-}<n>}: n \in \omega\right\}$-generated.

Let see first how such a construction implies the lemma. By definition of $T$ and conditions (a) and (b), $T$ is $1-1$. By condition (g) $F_{s}$ is not empty, hence by (c) and (d) $\bigcap_{n \in \omega} F_{f \mid n}$ contains exactly one element for every $f \in \omega^{\omega}$. But then by (f) this element is equal to $\varphi^{-1}(\{T(f \mid n): n \in \omega\})$, and hence $\{T(f \mid n): n \in \omega\} \in I$, which proves (i). 
We prove (ii) by induction on $n$. Assume that $B \in J$ and $B \subset \hat{s}_{0} \cup \cdots \cup \hat{s}_{k}$ for $\hat{s}_{0}, \ldots, \hat{s}_{k} \in \omega^{n}$. Then in particular $\{t \in B: \ln t<n\}$ is finite. Let $B^{\prime}=$ $B \backslash\{t \in B: \ln t<n\}$. Then, by definition of $T, T\left(B^{\prime} \cap \hat{s}_{i}\right) \in \mathscr{P}\left(A_{s_{i}} \backslash\left\{T\left(s_{i}\right)\right\}\right) \cap$ $I$. Hence by $(\mathrm{h})$ there is $k_{i}$ such that $T\left(B^{\prime} \cap \hat{s}_{i}\right) \subset A_{s_{i}^{-}<0>} \cup \cdots \cup A_{s_{i}^{-}<k_{i}>}$. It is easy to see that $\hat{t} \subset T^{-1}\left(A_{t}\right)$ for every $t \in \omega^{<\omega}$, so $B^{\prime} \subset \bigcup_{i \leq k}\left\{\hat{t}: t=s_{i}^{-}<m>\right.$ for $\left.m \leq k_{i}\right\}$, and we are done.

The construction: Assume we already constructed $F_{s}$ and $A_{s}$. Then by $(\mathrm{g})$, $\varphi\left(F_{s}\right)$ satisfies requirements of Lemma 1. Let $\left\{A_{n}: n \in \omega\right\}$ be as in Lemma 1 , and put $A_{s^{`}<n>}=\left(A_{s} \cap A_{n}\right) \backslash\{T(s)\}, F_{s^{\prime}<n>}^{\prime}=F_{s} \cap \varphi^{-1}\left(\mathscr{F}_{s^{\prime}<n>}\right)$. Then all conditions are satisfied, except perhaps (d). To make (d) true, split $F_{s^{\prime}<n>}^{\prime}$ into finitely many closed pieces of sufficiently small diameter. It is easy to see that at least one piece must satisfy $(\mathrm{g})$, and choose $F_{s^{-}<n>}$ to be such a piece.

We assume that the reader knows the concept of rank of well-founded subsets of $\omega^{<\omega}$. The key to the next lemma is the following classical characterization of analytic families of well-founded sets given by Lusin (see [K, Mo]).

Theorem 3 (Lusin). If a family $\mathscr{B}$ of well-founded subsets of $\omega^{<\omega}$ is $\Sigma_{1}^{1}$, then $\{\operatorname{rank}(B): B \in \mathscr{B}\}$ is bounded in $\omega_{1}$.

Lemma 3. If I an ideal on $\omega$ is $\Sigma_{1}^{1}$, bisequential and not countably I-generated then $\widehat{I} \notin \Sigma_{1}^{1}$.

Proof. Let $J$ be as in Lemma 2. Then by Lemma 2(i) every element of $\widehat{J}$ is well founded. We prove that for every $s \in \omega^{<\omega}$ the set $\{\operatorname{rank}(B): B \in \widehat{J} \cap \mathscr{P}(\hat{s})\}$ is unbounded in $\omega_{1}$. Assume that for every $s \in \omega^{<\omega}$ and every $\beta<\alpha<\omega_{1}$, we have $B_{s, \beta} \in \widehat{J} \cap \mathscr{P}(\hat{s})$ with $\operatorname{rank}\left(B_{s, \beta}\right) \geq \beta$. Let $\left\{\beta_{n}: n \in \omega\right\}$ be an enumeration of $\alpha$. Then by Lemma $2\left(\right.$ ii) $B=\bigcup_{n \in \omega} B_{s^{-}<n>, \beta_{n}} \cup\{s\} \in \widehat{J}$ and $\operatorname{rank}(B) \geq \alpha$. Hence, by the Lusin theorem $\widehat{J} \notin \Sigma_{1}^{1}$, but $\widehat{J}=T^{-1}(\widehat{I})$, so $\widehat{I} \notin \Sigma_{1}^{1}$.

\section{Results}

In this section we will derive Theorem 2 from the lemmas, and give some remarks.

Proof of Theorem 2. The implication from (i) to (ii) is easy. Indeed assume that $\left\{U_{n}: n \in \omega\right\}$ is a base of neighborhoods of $x$ for $Y$. Then

$$
\left\{\left(y_{i}\right) \in Y^{\omega}: \lim _{i \rightarrow \infty} y_{i}=x\right\}=\bigcap_{n \in \omega} \bigcup_{k \in \omega} \bigcap_{i \geq k}\left\{\left(y_{i}\right) \in Y^{\omega}: y_{i} \in U_{n}\right\}
$$

Since $\left\{\left(y_{i}\right) \in Y^{\omega}: y_{i} \in U_{n}\right\}$ is closed and open in $Y^{\omega}$, the set $\left\{\left(y_{i}\right) \in Y^{\omega}\right.$ : $\left.\lim _{i \rightarrow \infty} y_{i}=x\right\}$ is $\mathbf{F}_{\sigma \delta}$.

The implication from (ii) to (iii) is trivial.

To prove the implication from (iii) to (i), assume that $X \subset \mathbf{R}^{\omega^{\omega}}, x \in X$, $Y$ is a countable dense set in a neighborhood of $x$, and $Y$ has no countable base at $x$. Then $\{U \cap Y: U$ is an open neighborhood of $x\}$ generates a filter 
$F$ on $Y$. Since $Y$ is countable, we can assume that $F$ is a filter on $\omega$. Let $I=F^{*}$; since $Y$ has no countable base at $x, I$ is not countably $I$-generated . Moreover, by definition of the topology in $\mathbf{R}^{\omega^{\omega}}$,

$$
\begin{aligned}
A \in F \Leftrightarrow \exists f_{0}, \ldots, f_{n} \in \omega^{\omega} \exists a_{0}, \ldots, a_{n}, b_{0}, \ldots, b_{n} \in \mathbf{R} \forall i \leq n, \\
a_{i}<x\left(f_{i}\right)<b_{i} \wedge \forall y \in Y\left(\forall i \leq n a_{i}<y\left(f_{i}\right)<b_{i} \Rightarrow y \in A\right) .
\end{aligned}
$$

Since $X$ is Rosenthal, every element of $X$ has a Borel definition. Because $Y$ is countable the above is a $\Sigma_{1}^{1}$ definition of $F$. It is easy to see that, since $X$ is bisequential, the ideal $I$ is bisequential too. Hence $I$ satisfies the assumptions of Lemma 3.

Assume, for a contradiction that $\left\{\left(y_{i}\right) \in Y^{\omega}: \lim _{i \rightarrow \infty} y_{i}=x\right\} \in \Sigma_{1}^{1}$. Then $\left\{B \subset Y: \forall A \in F B \subset^{*} A\right\} \in \Sigma_{1}^{1}$, but this set is equal to $\widehat{I}=\{B \subset Y: \forall A \in$ $\left.I B \cap A={ }^{*} \emptyset\right\}$ and by Lemma $3, \widehat{I} \notin \Sigma_{1}^{1}$, a contradiction.

In fact, in the above proof of Theorem 2 we make use of a condition weaker then bisequentiality of $X$; all that we needed was a similar condition for one special ultrafilter in Lemma 2 . On the other hand, to keep the last arguments correct, every element of $X$ has to be analytic. We do not know whether there exists any compactum satisfying all that, but not a Rosenthal one.

Lemma 2 gives us more information. To make this precise consider two ideals on $\omega^{<\omega}, J_{0}=\left\{A: \exists f_{0}, \ldots, f_{n} \in \omega^{\omega} A \subset\left\{f_{i} \mid k: i \leq n, k \in \omega\right\}\right\}$ and $J_{1}=\left\{A: \exists f \in \omega^{\omega} A \subset\left\{s \in \omega^{<\omega}: \forall n<\operatorname{lh} s s(n)<f(n)\right\}\right\}$. In fact Lemma 2 says that $J_{0} \subset J \subset J_{1}$. Both ideals are $\Sigma_{1}^{1}$ and not countably generated, but $J_{0}$ is bisequential while $J_{1}$ is not. Nevertheless all the above, can be applied to $J_{1}$ (and its subideals) as well. $J_{1}$ is the maximal ideal satisfying the conclusion of Lemma 2. The minimal cardinality of a base of the ideal $J_{1}$ is usually denoted by $\mathbf{d}$. It is well known that both $\mathbf{d}=\omega_{1}<2^{\aleph_{0}}$ and $\omega_{1}<2^{\aleph_{0}}=\mathbf{d}$ are consistent with usual axioms of set theory. Moreover $\operatorname{cov}(\mathscr{K})=\{\min |\mathscr{F}|: \bigcup \mathscr{F}=\mathbf{R}$ and every element of $\mathscr{F}$ is meager $\} \leq \mathbf{d}$, and $\operatorname{cov}(\mathscr{K})<\mathbf{d}$ is consistent (see [Mi]). It was known that a Rosenthal compactum has at a given point either a countable base or no base of cardinality smaller than $\operatorname{cov}(\mathscr{K})$ (see [P2]). By Lemma 2 one can prove the following slightly stronger result.

Theorem 4. Let $X \subset \mathbf{R}^{\omega^{\omega}}$ be a Rosenthal compactum, $x \in X$ and $Y \subset X$ countable. Then either $Y$ has a countable base at $x$, or $Y$ has no base at $x$ of size less than $\mathbf{d}$.

The interesting open question is whether in the above $\mathbf{d}$ can be replaced by $2^{\aleph_{0}}$ (see [P2]).

\section{REFERENCES}

[BFT] J. Bourgain, D. H. Fremlin, and M. Talagrand, Pointwise compact sets of Baire measurable functions, Amer. J. Math. 100 (1978), 845-885.

[K] K. Kuratowski, Topology, Academic Press, New York, 1966.

[Ma] W. Marciszewski, On the classification of pointwise compact sets of the first Baire class functions, Fund. Math. 133 (1989), 165-209.

[Mi] A. W. Miller, Some properties of measure and category, Trans. Amer. Math. Soc. 266 (1981), 93-114. 
[Mo] Y. N. Moschovakis, Descriptive set theory, North-Holland, Amsterdam, 1979.

[P1] R. Pol, Note on pointwise convergence of sequences of analytic sets, Matematika 36 (1989), 290-300

[P2] On pointwise and weak topology in function spaces, preprint.

[R] H. P.Rosenthal, $A$ characterization of Banach spaces containing $l_{1}$, Proc. Nat. Acad. Sci. U.S.A. 71 (1974), 2411-2413.

University of Warsaw, Institut Matematyki, Banacha 2, 00-913 Warszawa 59, Poland 